\title{
Engineering Product-Service Solutions: An Application in the Power and Automation Industry
}

\author{
Giuditta Pezzotta ${ }^{1}$, Roberto Pinto ${ }^{1}$, Fabiana Pirola ${ }^{1}$, Sergio Cavalieri ${ }^{1}$, \\ Fumiya Akasaka $^{2}$, and Yoshiki Shimomura ${ }^{2}$ \\ ${ }^{1}$ CELS - University of Bergamo, Italy \\ \{giuditta.pezzotta, roberto.pinto, fabiana.pirola, \\ sergio.cavalieri\}@unibg.it \\ ${ }^{2}$ Department of System Design, Tokyo Metropolitan University, Japan \\ akasaka-fumiya@sd.tmu.ac.jp, yoshiki-shimomura@center.tmu.ac.jp
}

\begin{abstract}
Manufacturing companies are heavily competing for the identification of new sources of value for the market, moving from a traditional transaction-based and product-centric orientation towards the provision of integrated solutions to their customers. In this context, Service Engineering, the discipline concerned with the systematic development and design of service and productservices, is gaining particular relevance for mastering the service lifecycle management of a product-service solution. This paper provides a contribution in this field with the description of a Service Engineering Framework platform to support the reengineering of the service offering in a multinational company operating in the power and automation industry, with the aim to provide a customized solution able to fulfill the specific customer requirements and at the same time to gain a better efficiency in the delivery of the service processes.
\end{abstract}

Keywords: Service Engineering, Discrete-Event Simulation, Customer value.

\section{$1 \quad$ Introduction}

The recent macro-economic events have contributed to increase awareness of the strategic relevance deriving from the provision of services related to products, as an economic anti-cyclical remedy for tackling the dramatic contraction of the markets. This strategic evolutionary path followed by most manufacturing companies, usually termed as servitization, is mainly motivated by a continuous search of new sources of value, by either reactively fulfilling explicit requirements or proactively providing new integrated product-service solutions to the customer [1], evolving from a "pure product" orientation towards a more integrated product-service perspective [2].

One of the major managerial challenges is related to the need of companies to redesign their organisational principles, structures and processes [3], capabilities [4], relationships with customers [5] and suppliers [6]. In this sense, the design and development of a product-service solution and of its lifecycle raise new issues, since the service component introduces further requirements that in a traditional product-based business model are not relevant. In particular, the cultural shift from a transaction-based approach 
to a long-term relationship with a customer needs to be thoroughly understood by companies along with the acquisition of suitable models, methods and tools for collecting, engineering and embedding in a solution all the knowledge that meets or exceeds people's emotional needs and expectations. However, as reported by [1], even though a significant part of the literature has provided contributions on methods and tools, only a small number of approaches have been developed specifically for service and productservice design and engineering.

In such a context, Service Engineering (SE), calling for the design and the development of an integrated product-service offering valuable to customers and contributing to a continuous positive change of customer satisfaction and experience [7] [8], becomes a predominant field. The relevant aspect of SE as a new discipline is that it investigates product-service design and development considering both customer and company internal perspectives.

In spite of the SE advantages underlined by theory, only few authors have proposed methodologies and tools which can be easily adopted by industrial companies, that continue to maintain a product-centric focus during the design of a solution. In addition, the existing tools in SE are mainly focused on design solutions able to satisfy customer needs, without balancing the search for an excellent value provided to final customers with the achievement of better efficiency in the delivery of the service processes. Moreover, a Service Lifecycle Management (SLM) perspective is missed, reducing service to a mere delivery execution process. This could lead to the emergence of what has been defined as service paradox [9] as an evidence of the countereffects on the company's organization and its cost profile that could derive from venturing in these new markets due to a combination of hazard and opportunities.

In order to fill this gap, purpose of this paper is to discuss a real case application of the Service Engineering Framework which has been developed with the aim of integrating the design capabilities of the Service CAD, a modeling methodology for Service Design, with a simulation platform, enabling the comparison of several product-service configurations and the evaluation of technical and financial performances.

Using this framework, the service offering - in particular, the preventive maintenance service delivered by a multinational company operating on a global scale in the power and automation industry - has been investigated, with the purpose of providing re-engineering proposals of the current way it provides its services to the market. To illustrate the framework and its application, the paper is structured as follows. Section 2 is devoted to a literature review on SE with a brief state of the art of design and simulation methods and tools, focusing in particular on the Service CAD methodology and Discrete Event Simulation (DES). By making use of the case study, Section 3 provides an in-depth description of the single modules of the framework. Conclusions and further developments of the research are reported in the last section.

\section{$2 \quad$ Service Engineering}

The profit generation and the commercial success of a product-service solution critically depend on its conceptualisation, design and development, even if this notion has 
been largely ignored [7]. According to [10], the plethora of available tools and methodologies are typically a rearrangement of conventional processes, thus lacking a critical and in-depth evaluation of their performance in practice. Compared to physical products, services are generally under-designed and inefficiently developed [11]. Service Engineering (SE) is an emerging discipline aiming at filling this gap between product and service design. Sharing the definitions provided by [7] and [8], SE can be termed as a technical discipline concerned with a systematic development and design of services aiming at increasing the value of artefacts.

So far, a significant part of the SE literature has provided contributions on "HOW" a SE process must be carried out through the adoption of appropriate practices [7] [1], as well as methods and tools required to perform the single activities and phases. However, a small number of methods have been developed specifically for service design, development and engineering [1].

Among the different academic schools approaching the SE, Tokyo Metropolitan University has been working in this area since the beginning of the last decade developing the Service CAD methodology [12] [13] [14]. Service CAD is a modeling methodology created to describe and develop a customer value and its ground, the relationship between customer value and service contents, the service contents delivered by products and/or services [13]. The following paragraph better explains the main features of the Service CAD and the Service Explorer, a computerized design tool thought to describe and improve the quality of services.

\subsection{Basics of the Service CAD Methodology}

In accordance with the Service CAD methodology, a service is defined as an activity that changes the state of a service receiver by make him/her satisfied. More in detail, the receiver is described by a set of Receiver State Parameters (RSPs) representing the customer value [12].

The Service CAD provides some models which have to support a designer in the service design [12] [13], as briefly described in the following:

(1) the Flow model represents the flow among the various agents of a value chain.

(2) the View model describes, through a functional tree structure, the mutual relationships between the customer value, represented by a RSP, and the related functionalities. In the lower level of a View model the entities (hardware and humanware resources) and their attributes are introduced. Using a View model, designers perform a static evaluation of customer satisfaction based on these entities and their attributes.

(3) the Scope model describes the real activities taking place between two agents, i.e. between a receiver and a service provider. It deals with the RSPs of both the provider and the receiver, and handles multiple view models (namely, multiple RSPs).

(4) the Extended Service Blueprinting represents the service delivery process, and how the entities are connected with sub-processes and functions. The details of the relationships between functions and entities are here depicted. A Business Process Modeling Notation (BPMN) [18] is used in the extended service blueprint. 
Therefore, by connecting the service functionalities defined through the View model and the service delivery process defined by the Extended Service Blueprinting, designers can clarify the relationship between the service process and the customer expected value through the explication of its functionalities.

Based on the aforementioned modeling method, a computer-aided design system called Service Explorer has been under development since 2002 [14]. Aim of the Service Explorer is to describe the customer needs and their mutual relationships as well as to provide a design environment that can support designers from individual customer analyses up to entities construction.

\subsection{Discrete Event Simulation Models}

Simulation can be defined as "a technique of constructing a model that describes the behavior of a real world system, and the resulting model can then be used to test how the performance of a proposed system alters over differing operating conditions" [16]. Business process simulations have been extensively studied in the past, and many techniques and tools currently exist. In particular, Life Cycle simulation [17] and Discrete-Event Simulation (DES) modeling have been investigated in the area of product-service. The adoption of simulation in the service field is becoming relevant due to the increasing scale of operations, and to the complexity and the nature of operations connected. In this sense, simulation can be really supportive in the design of the service delivery process and in the evaluation of different systems created under multiple 'what-if' scenarios. Several existing projects aiming at modeling and simulating product-service systems are reported in literature [15].

In this research field, authors have mainly stressed the need to design solutions able to satisfy customer needs. However, in order to make service provision profitable in the long term, it is of utmost relevance to balance the excellence in the value channel to the customer with a high efficiency and productivity of the service processes, through the achievement of standardization and modularization of the underlying activities and resources. In this sense, the integration of Service CAD and simulation, and in particular DES, can really support managers to reach this equilibrium. The main advantage of DES is that the development of simulation models requires less assumptions, data and is more robust than analytical models.

\section{The Service Engineering Framework and Its Application}

The aim of the Service Engineering Framework is to support the engineering of a new product-related service and the reengineering of already available solutions. Further, it aims at allowing the definition of the most suitable and complete service and/or solution for a customer in terms of content and provision processes. As depicted in Fig. 1, the framework is based on five different modules, each one referring to a specific phase of the Product-Service Engineering process. The simulation model has been developed using the ARENA ${ }^{\circledR}$ simulation software by Rockwell Automation. 
Each phase is briefly presented hereafter. The description includes a general presentation of the main steps and the results obtained in the real case study application. The case refers to the application of the framework in a multinational company operating in the power and automation industry which is currently reengineering its service offering. The entire service portfolio has been analyzed and in particular the provision of industrial services based on preventive maintenance, identified as the most relevant service for the final customer, has been investigated.

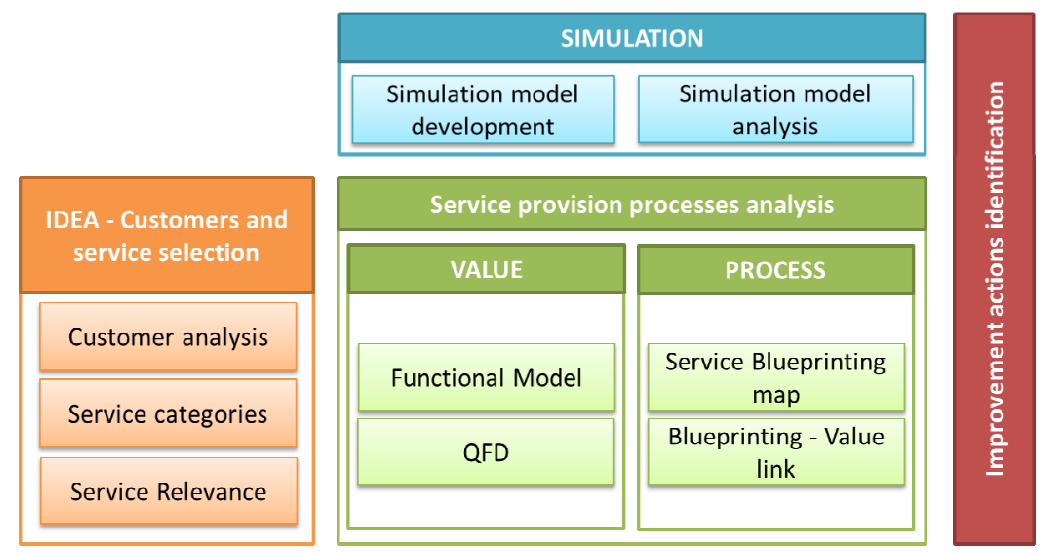

Fig. 1. The main modules of the proposed framework

\section{IDEA}

- Analysis of the customer segments based on their needs: by using the marketing data available (two surveys, one analyzing the customer needs and one the service preferences) two customer segments were identified: Seg.1) customers that rely on the producer's capability to maintain their equipment in good shapes; they want to be granted of an effective service execution; Seg.2) customers that would perform service activities autonomously, with the remote support of provider's technicians.

- Analysis of the service offering and needs satisfied through service provision: thanks to direct interviews to different Service Managers, a relationship between customer needs and services preferences was established; by matching customer needs with the needs fulfilled by services, the service offering that mostly suits the requirements of the two customer segments was identified.

- Identification of the most relevant services: four services were identified as relevant for both the customer segments; in particular, preventive maintenance was considered as the most strategic one; the aim is to satisfy customer's needs by providing a proper preventive maintenance service able to "maximize the customer's equipment availability".

\section{VALUE}

- Development of the functional tree: the functional tree of the need "maximize the customer equipment availability" was developed using the View model; this need was qualitatively decomposed into six functionalities at the first level; each of them 
was further drilled down until the definition of four detailed sub-levels of functionalities. At the last level, tangible entities and their attributes enabling to carry out the different functionalities were identified. This tree represents all the functionalities the service offer has to provide to the customer in order to satisfy his need. At the first level of the tree, among the six functionalities identified, the functionality "ensure failure prevention" was identified (Fig. 2). This functionality and the ones deriving from it represent the functional branch strictly related to the Preventive Maintenance service.

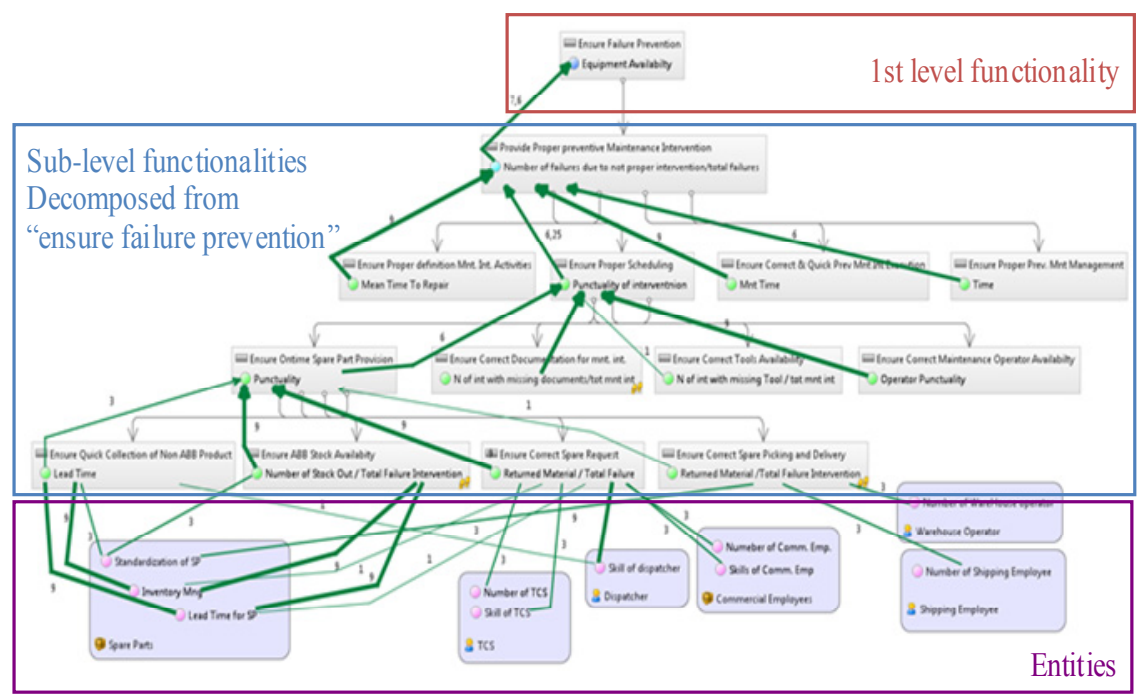

Fig. 2. The functional tree resulting from "ensure a proper failure prevention" functionality

- Analysis of the functionalities and entities relevance by use of QFD: Quality Function Deployment was adopted with the aim to identify the most relevant functionalities and entities of the preventive maintenance service the company can leverage on to optimize its provision process and satisfy customer needs. All the functionalities directly connected with the onsite execution of the maintenance and the human entities that have a direct relationship with the customer (e.g. maintenance operators and dispatcher) are the most critical ones to properly satisfy customer's needs.

\section{PROCESSS}

- Definition of the process blueprinting: the onsite and offsite preventive maintenance processes were modeled by use of the service blueprinting methodology. A detailed model of the processes was defined, and for each humanware entity a swimming lane in the process maps was reserved with the aim to better explain the process flow.

- Link of the blueprinting and the functionalities: in order to verify whether the current processes are able to satisfy the customer's need, it is fundamental to ascertain whether the functionalities identified in the previous phases are delivered along the 
process and which part of the process is the most critical in terms of customer satisfaction. The processes under analysis were able to provide the functionalities characterizing a good preventive maintenance service. Moreover, it was observed that the company reserves a lot of attention to the front-line activities.

\section{SIMULATION}

- Development of the simulation model: The static models developed in the Value and Process phases were transposed in the DES simulation tool environment. The transposition has been done manually by the translation of the BPMN model in the ARENA $^{\circledR}$ nomenclature. In particular, both the customer process and the service delivery processes (as detailed in the blueprinting) have been simulated by using real field data, and different scenarios were tested.

- What-if analysis of the simulation model: The scenarios were defined and evaluated based on the functional tree and the QFD analysis by a modification of: i) the saturation of the most relevant entities (maintenance operators and dispatcher) and ii) the priority assigned to the different functionalities and therefore to the different part of the process. Moreover, tests connected to a variation of the number of maintenance interventions were also carried out. In this way it was possible to identify the best solution which maximizes the expected performance, in terms of both company's revenues and customer satisfaction.

\section{Conclusions}

This paper presents the application of the Service Engineering Framework to support the re-engineering of a product-related service in a real company. Built around five different phases, the framework extends the logic of the Service CAD methodology through the introduction of a Discrete Event Simulation phase to support the economical and risk assessment of the most suitable product-service solution and the best performing provision process. Many are the advantages achievable through the introduction of this framework in the investigated company: (i) the adoption of a systematic procedure to analyse the existing services, (ii) an improvement of the process performance by the identification of the elements affecting customer values, (iii) a reduction of the costs and (iv) a better definition of the process changes in order to properly manage an increase of demand and revenues.

The output resulting from the simulation gave the company a lot of insights to improve its processes and to define a more customer-oriented structure. Due to confidentiality reasons, it was not possible to report in this paper more detailed data.

In order to better understand the appropriateness and robustness of the framework, further applications should be carried out either in engineering new services or in the reengineering of existing solutions enlarging also the perspective to non-industrial service systems, such as healthcare or touristic services.

As it was experienced along the case study, the main requirement for a fruitful adoption of the framework resides on the need of a massive quantity of field data which normally are not systematically collected and easily retrievable. 
Acknowledgments. The research leading to these results has received funding from the European Community's Seventh Framework Programme (FP7/2007-2013) under grant agreement $n^{\circ}$ PIRSES-GA-2010-269322. The paper has been also inspired by the activity of the ASAP SMF, an industry-academia community aimed at developing knowledge and innovation in product-services and service management (www.asapsmf.org).

\section{References}

1. Cavalieri, S., Pezzotta, G.: Product-Service Systems Engineering: State of the art and research challenges. Computers in Industry 63, 278-288 (2012)

2. Mont, O.K.: Clarifying the concept of product-service system. Journal of Cleaner Production 10, 237-245 (2002)

3. Gebauer, H., Fleisch, E.: An investigation of the relationship between behavioral processes, motivation, investments in the service business and service revenue. Industrial Marketing Management 36, 337-348 (2007)

4. Ceci, F., Masini, A.: Balancing specialized and generic capabilities in the provision of integrated solutions. Industrial and Corporate Change 20(1) (2011)

5. Galbraith, J.: Organizing to deliver solutions. Organizational Dynamics 31(2) (2002)

6. Evans, S., Partidário, P.J., Lambert, J.: Industrialization as a key element of sustainable product-service solutions. International Journal of Production Research 45 (2007)

7. Bullinger, H.J., Fahnrich, K.P., Meiren, T.: Service engineering-methodical development of new service products. International Journal Production Economics 85(3) (2003)

8. Shimomura, Y., Tomiyama, T.: Service modeling for service engineering. In: Arai, E., Fumihiko, K., Goossenaerts, J., Shirase, K. (eds.) Knowledge and Skill Chains in Engineering and Manufacturing, vol. 168, pp. 31-38. Springer (2005)

9. Gebauer, H., Fleisch, E., et al.: Overcoming the service paradox in manufacturing companies. European Management Journal 23(1), 14-26 (2005)

10. Baines, T.S., et al.: State-of-the-art in product-service systems. Journal Engineering Manufacture 221(B) (2007)

11. Froehle, C.M., Roth, A.V., Chase, R.B.: Antecedents of New Service Development Effectiveness: An Exploratory Examination of Strategic Operations Choices, North (2000)

12. Arai, T., Shimomura, Y.: Proposal of Service CAD System A Tool for Service Engineering. CIRP Annals - Manufacturing Technology 53(1), 397-400 (2004)

13. Hara, T., Arai, T., Shimomura, Y., Sakao, T.: Service CAD system to integrate product and human activity for total value. CIRP Journal of Manufacturing Science and Technology 1(4), 262-271 (2009)

14. Sakao, T., Shimomura, Y.: Service Engineering: a novel engineering discipline for producers to increase value combining service and product. Journal of Cleaner Production 15(6), 590-604 (2007)

15. Alix, T., Zacharewicz, G.: Product-service systems modelling and simulation as a strategic diagnosis tool. In: Emmanouilidis, C., Taisch, M., Kiritsis, D. (eds.) APMS 2012, Part II. IFIP AICT, vol. 398, pp. 361-368. Springer, Heidelberg (2013)

16. Baines, T., Mason, S., Siebers, P.O., Ladbrook, J.: Humans: the missing link in manufacturing simulation? Simulation Modelling Practice and Theory 12(7) (2004)

17. Garetti, M., Rosa, P., Terzi, S.: Life Cycle Simulation for the design of Product - Service Systems. Computers in Industry 63(4), 361-369 (2012)

18. Object Management Group: Business Process Model and Notation (January 2011), http : / / www . bpmn . org / 